BULLETIN Bulletin hispanique

HISPANIQUE Université Michel de Montaigne Bordeaux

117-2 | 2015

Métamorphose(s) : représentations et réécritures

\title{
La métamorphose de Narcisse
}

(Garcilaso de la Vega et Jean de la Croix)

\section{Suzy Béramis}

\section{(2) OpenEdition}

1 Journals

\section{Édition électronique}

URL : http://journals.openedition.org/bulletinhispanique/4068

DOI : 10.4000/bulletinhispanique.4068

ISSN : 1775-3821

Éditeur

Presses universitaires de Bordeaux

\section{Édition imprimée}

Date de publication : 15 décembre 2015

Pagination : $577-590$

ISBN : $979-10-300-0041-2$

ISSN : 0007-4640

Référence électronique

Suzy Béramis, "La métamorphose de Narcisse », Bulletin hispanique [En ligne], 117-2 | 2015, mis en

ligne le 15 décembre 2018, consulté le 23 avril 2019. URL : http://journals.openedition.org/

bulletinhispanique/4068; DOI : 10.4000/bulletinhispanique.4068 


\title{
La métamorphose de Narcisse (Garcilaso de la Vega et Jean de la Croix)
}

\author{
Suzy BÉRAMIS \\ Centre de Recherches sur l'Espagne du Siècle d'Or \\ Université Paris III
}

La deuxième églogue de Garcilaso offre une variation sur le mythe de Narcisse, qui associe la connaissance de soi à une expérience de l'amour si douloureuse qu'elle débouche sur la folie. Dans le Cantique Spirituel, Jean de la Croix reprend « a lo divino " cette même interrogation au miroir, née de l'absence de l'être aimé. Les deux poètes nous conduisent ainsi au seuil d'une métamorphose de l'homme qui concerne aussi bien l'âme que le corps.

Mots-clés : anthropologie, métamorphose, miroir, mysticisme.

La segunda égloga de Garcilaso ofrece una variación sobre el mito de Narciso, que asocia el conocimiento de sí a una experiencia del amor tan dolorosa que desemboca en la locura. En el Cántico Espiritual, Juan de la Cruz repite a lo divino esta misma interrogación al espejo, nacida de la ausencia del ser amado. Los dos poetas nos conducen así hasta una metamorfosis del hombre, que concierne tanto al alma como al cuerpo.

Palabras clave : antropología, metamorfosis, espejo, misticismo.

Garcilaso's second eclogue provides us with a variation on the myth of Narcissus, in which self-knowledge is linked to such a painful experience of love that it leads to madness. In his "Cántico Espiritual», John of the Cross uses again that questioning to the mirror, which arises from the Beloved's absence. Both poets lead us towards a metamorphosis of the human being, that affects both soul and body.

Keywords : Anthropology, Metamorphosis, Mirror, Mysticism. 
T 'influence de la poÉsie de Garcilaso de la Vega sur celle de Jean de la Croix L n'est plus à démontrer. Cependant, dans les commentaires qu'il réalise à ses propres poèmes, Jean de la Croix lui-même fait une seule fois référence à Garcilaso. C'est au début du traité de la Llama de Amor Viva, lorsqu'il présente la lira, la strophe qu'il a choisie pour exprimer les expériences les plus hautes de l'union de l'âme avec Dieu :

La compostura de estas liras son como aquellas que en Boscán están vueltas a lo divino, que dicen: La soledad siguiendo, I llorando mi fortuna, I me voy por los caminos que se ofrecen, etc., en las cuales hay seis pies, y el cuarto suena con el primero, y el quinto con el segundo, y el sexto con el tercero ${ }^{1}$.

Encore cette référence est-elle indirecte, car le nom même de Garcilaso n'y apparaît pas. Selon un usage très répandu à son époque, en effet, Jean de la Croix dit "Boscán » pour désigner le livre où étaient publiées ensemble les œuvres des deux amis, Juan Boscán et Garcilaso² ${ }^{2}$ Référence indirecte également parce qu'il cite le poème dans la version a lo divino de Sebastián de Córdoba, publiée en 1575, dans le but de canaliser cette poésie profane, qui exerçait sur tous une indéniable - et donc dangereuse - séduction, selon une spiritualité plus en accord avec les exigences du Concile de Trente ${ }^{3}$. Dámaso Alonso, dans La poesía de San Juan de la Cruz $z^{4}$, où il enquête sur les sources du poète mystique, n'a pas de mot assez dur pour qualifier une telle entreprise, et il exprime une inquiétude rétrospective à l'idée que celui-ci aurait pu connaître la poésie de Garcilaso uniquement à travers cet intermédiaire, versificateur laborieux, au moralisme trop étriqué selon lui. On ressent son soulagement de pouvoir prouver que, si Jean de la Croix connaît bien cette refonte a lo divino, il a, par ailleurs, vraiment lu Garcilaso lui-même, tout particulièrement la seconde Églogue, dont on retrouve l'influence dans la Llama de amor viva et le Cántico espiritual 5 .

Dans l'analyse que nous proposons ici, nous voudrions montrer que Jean de la Croix ne se contente pas de quelques emprunts formels, mais dialogue en profondeur avec cette œuvre profane, en particulier autour du thème de la métamorphose de Narcisse. Et dans ce dialogue, Sébastián de Córdoba joue un rôle précieux de médiateur, qu'il nous semble important de ne pas négliger trop vite.

1. Juan de la Cruz, Llama de Amor Viva, dans Obras Completas de san Juan de la Cruz, Madrid, Biblioteca de Autores Cristianos, 2009, edición crítica de Lucinio Ruano de la Iglesia, p. 915.

2. "Un Boscán" significaba, comercialmente y vulgarmente, "las obras de Boscán y Garcilaso" ". Dámaso Alonso, La poesía de san Juan de la Cruz (Desde esta ladera), Madrid, Aguilar, 1966, p. 39.

3. Sebastián de Córdoba, Garcilaso a lo divino, Madrid, Castalia, 1971. Edición crítica de Glen R. Gale. Le titre exact de la première édition (1575) est : Las Obras de Boscán y Garcilaso trasladadas a materias cristianas y religiosas.

4. Dámaso Alonso, op.cit., p. 37-41.

5. Ibid., p. 31-37. 
C'est donc cette seconde églogue, dans laquelle le pasteur Albanio, comme Narcisse, se penche sur le miroir de l'eau, qui va maintenant nous retenir. Ce long poème offre en effet une réélaboration du mythe d'Ovide, dont Jean de la Croix s'inspire dans le Cántico Espiritual.

Dans la deuxième Églogue de Garcilaso, le lieu central de l'action est la fontaine devant laquelle Albanio vient épancher sa peine d'amour à cause du dédain de Camila, sa compagne de jeux depuis l'enfance. C'est là que son ami Salicio le retrouve endormi et obtient qu'il lui raconte son histoire, là aussi qu'Albanio a révélé son amour à Camila. En lui demandant de se pencher sur l'eau pour y découvrir le visage de celle qu'il aime, il a provoqué la fuite de Camila, ce qui l'a conduit à une première tentative de suicide. C'est encore penchée sur cette eau que l'on retrouve Camila, un peu plus loin dans le poème, où elle se souvient de la révélation d'Albanio, à la fois peinée d'avoir perdu un ami aussi proche qu'un frère, et indifférente à sa souffrance. Consacrée à Diane, elle a fait vœu de virginité et, comme la déesse, sa principale activité est la chasse. C'est d'ailleurs en poursuivant un cerf qu'elle a blessé qu'elle est arrivée devant la fontaine. Fatiguée par la chasse, elle s'endort à son tour au bord de l'eau, comme Albanio plus haut. Celui-ci survient alors et la découvre endormie. Il se penche sur elle, la contemplant un instant dans son sommeil, puis lui saisit la main en un geste qui la réveille et provoque sa peur. Effrayée de se retrouver ainsi prisonnière, Camila réussit par ruse à obtenir qu'il accepte de la relâcher, et elle s'enfuit aussitôt, pour toujours. Albanio, qui se sent trahi, devient fou de désespoir et tente à nouveau de se suicider, en se laissant violemment tomber sur le sol. Il espère réussir de cette manière à sortir de son corps, délivrant ainsi son âme trop douloureuse :

\section{Recibe tú, terreno y duro suelo, este rebelde cuerpo, que detiene del alma el espedido y leve vuelo.}

Dans sa folie, qui exprime la fureur d'amour qui le domine - l'amor hereos il a le sentiment d'y être parvenu. Esprit désormais dépourvu de chair, il flotte, avec une légèreté telle qu'il lui semble voler :

\section{Descargado me siento de un gran peso; paréceme que vuelo, despreciando monte, choza, ganado, leche y queso ${ }^{7}$.}

De la hauteur où il se trouve, il aperçoit ses pieds très loin en bas. On a l'impression d'une sortie de soi, à la manière de l'extase dont parlent les

\footnotetext{
6. Garcilaso de la Vega, Poesía castellana completa, Madrid, Cátedra, 2003, "Égloga Segunda",

7. Ibid., v. 886-888.
} v. $874-876$. 
mystiques, que cependant l'esprit - celui d'Albanio - vit mal. Il parle en effet d'un corps volé. Il se sent dépossédé de lui-même et cherche son corps. Quand l'a-t-il perdu ? Sans doute, pense-t-il, pendant qu'il était occupé à regarder " autre chose ${ }^{8}$. Il se souvient d'une jolie forme endormie comme une rose, mais écarte aussitôt l'idée que ce soit son corps, car elle était trop belle. On reconnaît Camila qu'il a contemplée dans son sommeil. Poursuivant donc sa recherche - sa quête du corps - il se tourne à nouveau vers la fontaine, et y découvre une figure qui lui ressemble :

\section{Allá dentro en lo fondo está un mancebo de laurel coronado, y en la mano un palo propio, como yo, de acebo?.}

Comme le Narcisse d'Ovide, Albanio prend d'abord son reflet pour un autre, puis se reconnaît lui-même. Plus exactement, il reconnaît son corps qui s'est séparé de lui, et le presse de revenir :

$$
\begin{aligned}
& \text { ¿Oh cuerpo! Hete hallado, y no le creo; } \\
& \text { tanto sin ti me hallo descontento. } \\
& \text { Pon fin ya a tu destierro y mi deseo }{ }^{10} \text {. }
\end{aligned}
$$

Son corps est comme un autre, silencieux, qui semble ne pas pouvoir revenir :

Si no estás en cadenas, sal ya fuera a darme verdadera forma de hombre, que agora sólo el nombre me ha quedado ${ }^{11}$.

Dans sa douleur, cette forme penchée sur le miroir, esprit qui cherche son corps, n'est plus seulement Narcisse. Il devient Orphée, qui veut partir récupérer son corps comme le poète de Thrace à la recherche d'Eurydice :

$$
\begin{aligned}
& \text { [...] si al cielo que me oyere, } \\
& \text { con quejas no moviere y llanto tierno, } \\
& \text { convocaré el infierno y reino escuro, } \\
& \text { y romperé su muro de diamante, } \\
& \text { como hizo el amante blandamente } \\
& \text { por la consorte ausente, que cantando } \\
& \text { estuvo halagando las culebras } \\
& \text { de las hermanas negras mal peinadas }
\end{aligned}
$$

Dans ce poème, trois histoires se rencontrent, comme si l'une éclairait l'autre et pouvait en révéler la signification profonde, trois situations devant le
8. Ibid., v. 893.
9. Ibid., v. 913-915.
10. Ibid., v. 925-927.
11. Ibid., v. 934-936.
12. Ibid., v. 938-945. 
miroir. Et cette silhouette qui se penche sur son reflet - Narcisse, Albanio ou Orphée - exprime toutes les séparations du miroir. Des miroirs. L'homme et la femme, l'homme et son âme, le corps et l'esprit. Et, quand on regarde vers le ciel muet, l'homme et Dieu. Deux amis d'Albanio, Salicio et Nemoroso, assistent au spectacle de cette folie, et ils restent pensifs. Que de propos sensés dans ce discours de fou ! Le ton devient sérieux soudain, et Albanio, devant le miroir, atteint à une grandeur tragique : ce qui lui arrive dépasse le drame de sa rupture avec Camila. Il pose les questions que chacun se pose. Et les deux autres bergers reconnaissent, dans son étrange monologue, des préoccupations qui leur sont familières.

Nous allons nous arrêter à cette scène, parce que le poème nous y invite. La réflexion des deux amis suspend un instant le cours de l'églogue, avant qu'Albanio ne soit repris d'un accès plus violent de folie. Salicio et Nemoroso sentent alors l'urgence d'intervenir et Albanio, maîtrisé, s'endort de nouveau près de la fontaine, comme au début de l'églogue.

Cette forme cyclique enserre la première partie de l'églogue dans un cadre parfait, comme celui d'un tableau, dans lequel Salicio et Nemoroso jouent le rôle de "l'admoniteur " d'Alberti, c'est-à-dire ce personnage qui, dans le tableau, " attire l'attention des spectateurs sur ce qui se passe, de la main appelle le regard [...] ou indique là quelque danger ou quelque chose à admirer ${ }^{13}$. Ils nous signalent qu'à travers sa folie Albanio aborde des questions essentielles, mais le texte n'en dira pas plus. C'est une situation qui stimule le lecteur à approfondir le sens de l'œuvre. Mais quelles clés lui sont données?

Très certainement le mythe lui-même, qui vient d'être désigné. Le poème nous invite à lire l'histoire d'Orphée à travers celle de Narcisse, dans une approche où toutes les directions s'ouvrent à l'interprétation. Mais il ne peut s'agir d'une lecture suivie, car la deuxième partie de l'églogue semble tellement étrangère à l'univers de la folie d'Albanio, au moins dans une première approche, que c'est ailleurs, dans l'œuvre de Garcilaso, que nous allons poursuivre notre recherche sur ce Narcisse, qui se laisse identifier comme Orphée, tout en refusant de se confondre complètement avec celui-ci, car c'est lui-même qu'il cherche en cherchant son corps volé, et non, comme il l'a dit plus haut "otra cosa " ${ }^{14}$. Le jugement énigmatique que portent ses deux amis, reconnaissant dans les propos d'Albanio leurs propres interrogations, nous permet en effet de sortir du cadre de ce poème, pour reprendre, à travers d'autres textes, le dialogue avec leur auteur. Et c'est dans la troisième églogue (v. 121-144) que nous retrouvons l'histoire

13. Leon Battista Alberti, De la Peinture, Paris, Éditions du Seuil, éd. T. Golsenne et B. Prévost, 2004, p. 149.

14. Égloga Segunda, v. 893. Dans un autre poème - le sonnet XV - où la voix poétique compare sa peine de vivre à celle d'Orphée, comme ici Albanio, Garcilaso utilisera cette même expression pour dire que la perte de soi est une souffrance plus grande que celle qu'on éprouve à cause de l'autre qu'on aime :

"Con más piedad debría ser escuchada

la voz del que se llora por perdido

que la del que perdió y llora otra cosa" (v. 12-14). 
d'Orphée et d'Eurydice, tissée par l'une des nymphes, sorties des profondeurs de l'eau pour s'installer sur la rive du Tage. En une longue ekphrasis, le poème décrit cette toile, puis celles des trois autres nymphes, qui représentent l'histoire d'Apollon et de Daphné (v. 145-168), de Vénus et d'Adonis (v. 169-192) et la lamentation funèbre pour la belle nymphe Elisa (v. 193-264), dans laquelle on reconnaît Isabel Freyre dont le poète Garcilaso pleure la mort. Cette dernière scène, plus longuement décrite, s'introduit dans l'univers mythologique des trois premières pour évoquer la perte de l'être aimé, et la souffrance d'un deuil impossible.

De toutes ces histoires qui se rencontrent, émerge une interrogation sur la mort, dont Orphée réussit à franchir le seuil, même s'il ne parvient pas à ramener Eurydice. Sur la métamorphose aussi, à travers celle de Daphné qui se fige en arbre dans les bras d'Apollon désespéré15. Chef-d'œuvre d'imitation, le tissage des nymphes redonne une vie illusoire à ces ombres enfuies, ces formes vaines que l'on voudrait saisir ${ }^{16}$.

La troisième églogue se termine sur une note plus légère, le chant amébée de Tirreno et Alcino, deux bergers qui chantent à l'envi les louanges contrastées de leurs bien-aimées. Les nymphes écoutent sans se montrer, tandis que le soir tombe. Leur présence silencieuse nous entraîne vers le sonnet XI, où la voix poétique les appelle à écouter son chant :

\section{Hermosas ninfas, que en el río metidas contentas habitáis en las moradas de relucientes piedras fabricadas $y$ en colunas de vidrio sostenidas ${ }^{17}[\ldots]$.}

Ces vers font écho au chant d'Aristée, dans les Géorgiques (IV), lorsque celuici, désespéré d'avoir perdu ses abeilles, vient supplier sa mère, la néréide Cyréné, de l'aider. Dans le poème de Virgile, Cyréné est « au fond de sa chambre dans les profondeurs du fleuve $»^{18}$, entourée par des nymphes qui tissent et filent, comme celles que nous présentent la troisième églogue de Garcilaso mais aussi la strophe suivante de ce même sonnet ${ }^{19}$. C'est alors qu'elles entendent la plainte d'Aristée : «Toutes, sur leurs sièges de cristal, restèrent interdites ; mais plus prompte que toutes ses sœurs, Aréthuse, éleva sa tête blonde au-dessus des ondes $»^{20}$.

15. "Llora el amante, y busca el ser primero, besando y abrazando aquel madero." (v. 167-168).

16. Ibid., v. 265-272.

17. Garcilaso de la Vega, Poesía castellana completa, Madrid, Cátedra, 2003, "Soneto XI " (v. 1-4).

18. Virgile, Géorgiques IV, 333. Paris, Les Belles Lettres, 1995. Texte établi et traduit par E. de Saint-Denis, $9^{\mathrm{e}}$ tirage revu et corrigé par R. Lesueur, p. 69.

19. Garcilaso de la Vega, op. cit., v. 5-6.

20. Géorgiques IV, 350. Garcilaso reprend cette jolie image dans la troisième églogue, lorsque les nymphes sortent de l'eau pour s'installer au bord du Tage :

"Peinando sus cabellos de oro fino, 
Elle reconnaît Aristée, et les nymphes le conduisent à sa mère, qui lui révèle que seul Protée, le dieu marin, peut lui expliquer la cause de la mort de ses abeilles, " car, devin, il sait tout, le présent, le passé, la longue suite des faits à venir $»^{21}$. Mais il faudra pour cela qu'Aristée réussisse à l'approcher en profitant de son sommeil, et à l'enchaîner. Alors Protée ne cessera de changer de forme, multipliant les métamorphoses pour lui échapper :

Lorsque tes mains l'auront saisi et que tu le tiendras garrotté, alors il voudra se jouer de toi en prenant des apparences changeantes et même des figures de bêtes : en effet, il se fera soudain porc hérissé, tigre affreux, dragon écailleux et lionne à la nuque fauve ; ou bien il fera entendre le pétillement vif de la flamme, et cherchera de cette façon à s'échapper des liens, ou bien, se dissolvant en minces filets d'eau, à disparaître. Mais plus il multipliera ses métamorphoses, plus tu devras, mon fils, resserrer l'étreinte des liens, jusqu'à ce qu'il reparaisse, après transformation, tel que tu l'auras vu, quand il fermait les yeux dans son premier sommeil ${ }^{22}$.

Aristée réussit l'épreuve, forçant ainsi Protée à lui révéler la cause de la perte de ses abeilles : il s'agit d'un châtiment divin, parce que c'est Aristée qui, en poursuivant Eurydice, a provoqué sa mort et la douleur d'Orphée. Ayant obtenu cette réponse, Aristée revient auprès de sa mère, qui lui fait accomplir un rite expiatoire, au cours duquel il sacrifie quatre taureaux et autant de génisses afin d'apaiser les mânes d'Orphée et d'honorer Eurydice. Et neuf jours plus tard, "prodige soudain et merveilleux à dire, on voit, à travers les chairs liquéfiées des bœufs, des abeilles grouiller dans tout leur ventre en bourdonnant et s'échapper à gros bouillons des flancs éclatés, puis se former en nuées immenses et affluer en masse au sommet d'un arbre dont elles font ployer les branches en y suspendant leur grappe $»^{23}$.

Ce retour des abeilles, présenté ici comme un événement merveilleux qui survient au terme d'une initiation, caractérise une naissance qui se produit au sein même de la mort et de la corruption. Ovide l'évoque, lui, comme un fait d'expérience, au livre XV des Métamorphoses, où il donne la parole à Pythagore :

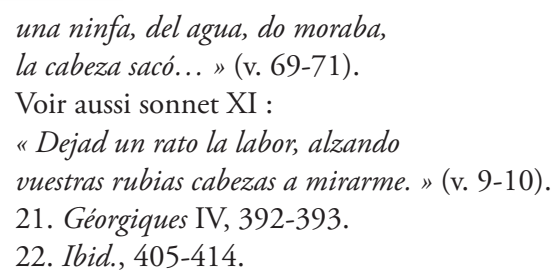

Aristée maitrisant Protée pendant ses métamorphoses symbolise celui qui a pénétré le mystère des choses et les transformations de la matière du monde : "Protée incarne le paradoxe d'un univers inquiétant, labile, changeant, "protéiforme", qui se place sous le signe de la métamorphose, de la ruse et de l'illusion, mais aussi de la vérité prophétique dont le héros en quête de sagesse doit s'emparer dans la violence et par la contrainte ", Anne Rolet, "Avantpropos ", dans Anne Rolet (sous la dir. de) Protée en trompe-l'ail. Genèse et survivances d'un mythe, d'Homère à Bouchardon, Presses Universitaires de Rennes, Collection "Interférences ", 2009 , p. 9.

23. Géorgiques IV, 552. 
Cependant s'il faut ajouter foi aux faits bien établis, ne voyez-vous pas des corps, que l'action du temps ou de la chaleur a fondus et décomposés, se transformer en petits animaux ? Tenez : choisissez une fosse, immolez-y des taureaux et rejetez sur eux de la terre ; par un phénomène que l'expérience atteste, de leurs chairs en putréfaction naissent çà et là des abeilles qui vont butiner les fleurs. ${ }^{24}$

Dans la troisième églogue de Garcilaso, tandis que les nymphes tissent leurs toiles, un bourdonnement d'abeilles accompagne leur travail :

En el silencio sólo se escuchaba

un susurro de abejas que sonaba ${ }^{25}$.

María Rosa Lida de Malkiel, dans un article où elle étudie ce motif poétique dans la littérature de la Renaissance, le met en relation avec l'évocation de l'Âge d'Or ${ }^{26}$, ce temps heureux où, comme le dit don Quichotte dans son discours aux chevriers, "En las quiebras de las peñas y en el hueco de los árboles formaban su república las solicitas y discretas abejas, ofreciendo a cualquiera mano, sin interés alguno, la fértil cosecha de su dulcísimo trabajo ". Mais dans l'églogue de Garcilaso, les nymphes sorties des profondeurs de l'eau, et cette toile qui raconte l'histoire d'Eurydice et d'Orphée, sont une claire référence à la fable d'Aristée. L'abeille symbolise la métamorphose ; et à travers les échos qu'il crée d'un mythe à l'autre, d'un auteur à l'autre - Ovide et Virgile -, Garcilaso s'interroge sur la mort et la renaissance espérée, pour lui-même et pour l'être aimé. Il s'interroge aussi sur les frontières des mondes, et l'origine de toutes choses, sur ce qui change et se transforme. Ces références, le plus souvent implicites, sont plus faciles à déchiffrer pour les lecteurs de son époque qu'aujourd'hui, mais nous restons sensibles à l'angoisse d'Albanio, dont la folie nous révèle un être dissocié, qui se cherche lui-même, à travers le manque douloureux que l'expérience amoureuse a creusé. Pour lui, la première métamorphose sera de reconquérir sa propre unité.

Garcilaso pose les questions et ne semble pas y apporter de réponse, sinon par cette guérison miraculeuse que promet la seconde partie de l'Églogue, où les amis d'Albanio se préparent à le conduire chez le mage Severo. Celui-ci, instruit par les dieux des secrets de la nature, est investi de pouvoirs merveilleux ${ }^{27}$. Mais à partir d'ici, le poème se présente surtout comme un long panégyrique à la gloire du duc d'Albe, le mécène de Garcilaso. Nous allons donc en interrompre la lecture, pour examiner ce même thème de la métamorphose avec Jean de la Croix.

24. Ovide, Les Métamorphoses, Paris, Les Belles Lettres, 2002. Texte établi et traduit par Georges Lafaye. Troisième tirage de l'édition revue et corrigée par Henri Le Bonnie, t. III, p. 133 (Livre XV, v. 361-367).

25. Égloga tercera, v. 79-80.

26. María Rosa Lida de Malkiel, "La abeja: historia de un motivo poético ", Romance Philology, no 17 (1), Aug. 1963, p. 75.

27. "A aquéste, Febo, no le escondió nada; antes de piedras, hierbas y animales

diz que le fue noticia entera dada " (v. 1074-1076). 
Quoique Narcisse joue un rôle important dans le Cántico espiritual, la présence du mythe y demeure en quelque sorte voilée, car Jean de la Croix n'en parle pas une seule fois de façon explicite. Pour éclairer les réalités de l'expérience mystique, sa préférence va à des modèles bibliques : Tobie, Jonas, Moïse ou Élie et, bien évidemment, le Cantique des Cantiques. Le monde de la mythologie n'est jamais évoqué, et Narcisse semble absent. On le reconnaît pourtant à la strophe XI du poème, lorsque l'âme amoureuse, qui cherche Dieu, se penche à son tour sur l'eau de la fontaine :

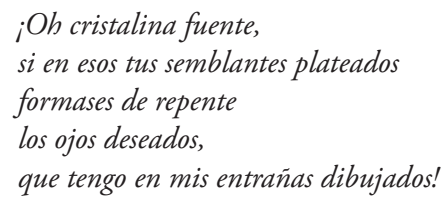

Dans le commentaire du poète lui-même à cette strophe, la référence majeure est le miroir paulinien de la Première Épitre aux Corinthiens ${ }^{28}$, mais les influences se mêlent et, quoique discrète, celle de Garcilaso est bien réelle dans le Cántico Espiritual, au moins jusqu'aux strophes 13-14A (14-15B) - les strophes de l'extase - où "nemoroso ", ce nom si familier aux lecteurs de Garcilaso, devient un adjectif qui exprime la beauté du monde contemplée dans un miroir qui est l'être même de Dieu :
Mi Amado las montañas,
los valles solitarios nemorosos,
las insulas extrañas,
el silbo de los aires amorosos ${ }^{29}[\ldots]$.

Par ailleurs, nous voudrions également mettre en évidence le parti que tire Jean de la Croix du Garcilaso a lo divino de Sebastián de Córdoba, qui joue un rôle important d'intermédiaire. Car il est évident que Jean de la Croix a apprécié cette œuvre, pour des raisons sans doute étrangères à toute considération esthétique ou purement littéraire. Il s'inspire, en particulier, de son système de personnages. Avant de commencer sa reprise de la deuxième églogue, Sebastián de Córdoba précise en effet :

Esta égloga, en la cual Garcilasso de la Vega pone un pastor llamado Albanio, aqui se llama Silvano por la parte sensual del hombre, y donde allá se llama otro pastor Salicio,

28. I Corinthiens XIII, 12 : «À présent nous voyons dans un miroir et de façon confuse, mais alors, ce sera face à face. À présent, ma connaissance est limitée, alors je connaîtrai comme je suis connu ". La Bible, Traduction CEcuménique de la Bible. Site de l'Alliance Biblique Française [en ligne]. Disponible sur http:// www. la-bible.net.

29. Juan de la Cruz, Cántico Espiritual, 13A (14B). Dans Obras Completas de san Juan de la Cruz, Madrid, Biblioteca de Autores Cristianos, 2009, edición crítica de Lucinio Ruano de la Iglesia. 
aqui se llama Racinio, por la razón, y la pastora que allá le dize Camilla, aqui se llama Celia, que es el alma, y el pastor Nemoroso se llama aqui Gracioso, por la gracia, con cuya fuerça el hombre vence a sí mismo ${ }^{30}$.

Ces figures allégoriques rappellent les mystères médiévaux ou el auto sacramental. Mais elles offrent une précieuse clé d'interprétation, puisqu'on voit se déployer, à travers les personnages de l'églogue, les diverses composantes de l'être humain : l'âme et le corps, l'esprit... Jean de la Croix s'en souviendra dans sa propre relecture du poème de Garcilaso, mais la rendra plus complexe. Car ce qui est en jeu, dans l'expérience mystique, quand l'âme parvient au stade du miroir, c'est précisément cette image dissociée dont il faut reconstituer l'harmonie. Le Silvano de Sebastián de Córdoba - c'est-à-dire la partie sensuelle de l'homme - a vu Celia - l'âme - s'éloigner de lui, et finir par le fuir, parce qu'elle ne veut plus partager avec lui des désirs grossiers et des aspirations qui la souillent et la dégradent. Elle veut désormais vivre son propre désir, qui la tourne vers Dieu.

Celia rappelle l'âme amoureuse qui, au début du Cántico Espiritual, se plaint de l'absence de l'Aimé. "Sortie d'elle-même et de toutes choses ", elle souffre de n'être pas délivrée de la chair mortelle afin de jouir de Dieu dans la gloire de l'éternitée ${ }^{31}$. Cette sortie de soi, c'est la fuite de Celia. Mais l'optique n'est plus celle, un peu lourdement moralisatrice, de Sebastián de Córdoba. Jean de la Croix décrit un stade déjà élevé de l'expérience mystique - l'amour impatient -, pendant lequel l'âme vit une relation difficile avec le corps. Celuici souffre lui aussi, mais le docteur mystique précise, dans le traité de la Noche Oscura, qu'elle n'en fait pas plus cas que l'arbre d'une de ses feuilles ${ }^{32}$. Cette étrange image évoque la métamorphose, et elle annonce une évolution à vivre, pour le corps et pour l'âme.

Mais Jean de la Croix s'inspire également de la Camila de Garcilaso. Son âme amoureuse est, elle aussi, une chasseresse. Au début du Cántico, elle aussi poursuit un cerf, mais c'est elle qui est blessée. Le poème mystique reprend ici un thème familier à la littérature courtoise, pour exprimer, dans l'amour, la réciprocité, et le processus d'identification. Car lorsque sera venu le temps de la rencontre, et des fiançailles, l'Aimé se montrera à elle, blessé lui aussi, « ciervo vulnerado", au sommet de la colline ${ }^{33}$.

La chasse, pour Camila qui sert Diane, la déesse de la chasteté, comme pour l'âme amoureuse du Cántico, a une dimension sacrée. Elle exprime une manière d'habiter le monde tous ses sens en éveil, attentif au moindre signe. Aux aguets, à l'affût, cherchant la trace. La chasse révèle la signification religieuse de la

30. Sebastián de Córdoba, Garcilaso a lo divino, p. 170.

31. Cántico Espiritual I, $\$ 2$.

32. "Se causa en el alma, por razón del Amado, un ordinario 'sufrir sin fatigarse’; [...] El espiritu aqui tiene tanta fuerza, que tiene tan sujeta a la carne y la tiene tan en poco como el árbol a una de sus hojas». Juan de la Cruz, Noche Oscura II, chap. 19, $\$ 4$. Dans Obras Completas de san Juan de la Cruz, Madrid, Biblioteca de Autores Cristianos, 2009, edición crítica de Lucinio Ruano de la Iglesia.

33. Cántico Espiritual, 12A (13B). 
présence de l'homme au monde. Et ce cerf qui fuit ouvre un ailleurs et un vide, qui créent en l'âme une souffrance intense, en même temps que savoureuse, parce que l'infini se révèle ainsi en Dieu qui se cache et qui se montre ${ }^{34}$. Les soudaines et fugitives apparitions du cerf sont comme des flèches qui atteignent le mystique en plein cœur, au centre vital, et il découvre le vivre agonique, cette douleur de mourir et renaître à chaque instant :

[Estas saetas] inflaman tanto la voluntad en afición, que se está el alma abrasando en fuego y llama de amor, tanto que parece consumirse en aquella llama, y la hace salir fuera de si y renovar toda y pasar a nueva manera de ser, asi como el ave fénix, que se quema y renace de nuevo. De lo cual hablando David (Ps 72, 21-22), dice: [...] Fue inflamado mi corazón y mis renes se mudaron, y yo fui resuelto en nada, y no supe $e^{35}$.

Une transformation est en train de se réaliser, viscérale, affective, spirituelle en même temps. Très douloureuse à cette étape de l'expérience spirituelle où les angoisses de l'amour et la peine extrême du désir de Dieu se confondent avec les dernières épreuves de la purification. La métamorphose, c'est-à-dire le processus de divinisation, est en cours ${ }^{36}$. L'âme sort des limites de l'expérience ordinaire, car Dieu l'attire à lui. Et c'est aussi comme cela qu'il faut comprendre la sortie : un douloureux arrachement à soi-même, que le mystique ressent d'une manière presque physique. Comme si l'on tirait l'âme du corps :

$Y$ asi, es como si dijera: "Esposo mío, en aquel toque tuyo y herida de amor [...] me hiciste salir de mi (porque, a la verdad, y aun de las carnes parece que entonces saca Dios al alma) y levantásteme a ti, clamando por $t i$, desasida ya de todo para asirme a ti" ${ }^{37}$.

On dirait que l'âme apprend à voler, et qu'elle découvre avec difficulté l'étrangeté de cette situation nouvelle entre le ciel et la terre. Elle cherche un appui qui lui fait maintenant défaut : " $Y$ "eras ido". Como si dijera: al tiempo que quise comprender tu presencia no te hallé, y quedéme vacía y desasida de todo, $y$ sin asirme a ti penando en los aires de amor, sin arrimo de ti y de mí $1^{38}$.

Ce vol encore mal assuré est l'image d'un dépassement de la condition humaine. Cela rappelle la sortie de soi d'Albanio, son impression de voler. Chez lui aussi une métamorphose est en cours, qui échappe aux catégories ordinaires. Mais l'expérience, mal canalisée, ouvre sur la folie. Le personnage de Garcilaso aura besoin du secours d'un grand initié, le mage Severo, pour obtenir la guérison. Son expérience semble proche de celle du mystique, à ceci près que, chez Jean de la Croix, c'est l'âme - Celia, ou Camila - qui est sortie. Non pas Albanio, ou le Silvano de Sebastián de Córdoba. Mais cela ouvre la porte à un dialogue entre les deux grands poètes, qui nous parlent, et se parlent, du corps et de l'âme.

34. Ibid., I, $₫ 15$.

35. Ibid., \$17.

36. "Estas se llaman heridas de amor, que son al alma sabrosísimas [...] porque la hacen salir de si y entrar en Dios ». Ibid., $\$ 19$.

37. Ibid., $\$ 20$.

38. Ibid., $\$ 21$. 
Avec Jean de la Croix, on découvre l'univers de Camila, cette chasse qui ne prendra jamais fin ici-bas, parce qu'elle exprime la relation à l'infini et au divin. Dans cette course, qui semble vide, Camila se réalise elle-même comme infinie. Elle accomplit avec le Dieu qu'elle poursuit, le cerf, une ressemblance qui demeure pourtant toujours un horizon à atteindre. Sa plénitude est de se vivre comme ce mouvement et cette quête sans fin, cette ouverture à l'invisible ${ }^{39}$. C'est pour cela qu'elle fuit son image dans le miroir. L'âme, dans son essence, doit fuir toujours. Camila ne doit pas revenir. Et Albanio doit consentir à cette étrange relation avec lui-même, faite de fuites et de retrouvailles, à l'image des apparitions et disparitions du cerf. L'homme devient lui-même, en s'échappant sans cesse, comme le rappellent ces vers du dessin du Monte Carmelo :

\section{Para venir a lo que no eres}

has de ir por donde no eres ${ }^{40}$.

Sans doute cette vision mystique de l'âme peut-elle expliquer quelque chose de la mystérieuse séduction qu'exerce le personnage de la mujer esquiva dans la littérature courtoise.

Par ailleurs, nous aimerions comparer l'expérience du " corps volé » dans le poème de Garcilaso, à celle du " cœur volé " dans le Cántico espiritual. C'est à la strophe 9 du poème, où l'âme, dont le désir est sans cesse frustré, adresse à l'Aimé de véhéments reproches :

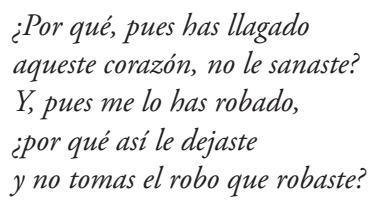

Dans cette strophe, "le vol " est une image paradoxale à travers laquelle Dieu apparaît comme celui qui transgresse et qui stimule ainsi, par la violence qu'il lui fait, à la fois le désir de l'âme et sa violence en retour, sa colère. Certes, le procédé est fréquent dans la rhétorique amoureuse, mais c'est en jouant sur les mots "robar / arrobar", dans le commentaire qui suit, que Jean de la Croix exprime l'idée que l'union entre l'âme et Dieu est une transgression. Sous l'expression raffinée des sentiments, inspirée de la littérature profane, il faut retrouver ce que l'expérience a d'insolite, et de radicalement nouveau : il s'agit, dans cette rencontre, de franchir une barrière, de se mettre " hors la loi ", car

39. Cf. ce passage du commentaire à propos des "insulas extrañas" qui, dans le poème, symbolisent l'être de Dieu : "Por las grandes y admirables novedades y noticias extrañas alejadas del conocimiento común que el alma ve en Dios, le llama "insulas extrañas". [...] Y no es maravilla que sea Dios extraño a los hombres que no le han visto, pues también lo es a los santos ángeles y almas que le ven; pues no le pueden acabar de ver ni acabarán. ", Cántico espiritual, 13A (14B) $\$ 8$.

40. On retrouve ces mêmes vers à la fin du premier livre de la Subida del Monte Carmelo, chap. $13, \$ 11$. Jean de la Croix précise là que le dessin du Monte Carmelo doit se trouver au début du traité et accompagner les différentes étapes de l'expérience spirituelle. 
le divin et l'humain sont, par nature, incompatibles. Mais le hors-la-loi, c'est Dieu. L'union est un rapt, au sens propre du terme, et Dieu est le voleur :

Robar no es otra cosa que desaposesionar del robo a su dueño y aposesionarse dello el robador. Esta querella, pues, propone aqui el alma al Amado, diciendo que, pues él ha robado su corazón y sacádolo de su poder y posesión, que ipor qué le ha dejado asi sin ponerle de veras en la suya, tomándolo para sí, como hace el robador al robo que robó, que de hecho se le lleva?

Por eso el que está enamorado se dice tener el corazón robado o arrobado de aquel a quien ama, porque le tiene fuera de sí, puesto en la cosa amada ${ }^{41}$.

Dieu est le voleur, mais l'âme est consentante, et ce qu'elle lui reproche, c'est de ne pas aller jusqu'au bout de son acte. Le cœur volé est resté à mi-chemin, " como el que está colgado en el aire, que no tiene en qué estribar " ${ }^{42}$. À l'étape de l'amour impatient, ce cœur sorti de sa place, soulevé au-dessus de lui-même, symbolise la purification des sentiments et des passions, des instincts. Cela veut dire que toutes les motivations du désir deviennent clairement conscientes pour l'âme qui veut se donner, mais aussi posséder ce qu'elle aime. L'amour impatient est comme une agonie, mais ce sont aussi les forces brutes de l'âme qui sont réveillées, les forces viscérales, qui se tournent vers Dieu avec une agressivité qui est aussi de l'amour :

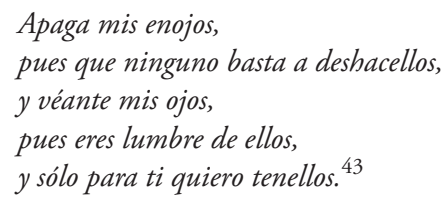

Ces " colères " sont toutes les forces instinctives de l'âme qui se soulèvent. Elles la fatiguent et l'épuisent, et le désir qui les nourrit est comme un feu démesuré que rien ne peut apaiser. Mais en réalité, et sans que l'âme en soit immédiatement consciente, une reconstruction est en cours en elle, en même temps qu'une mise à mort. Dieu est en train de transformer ses énergies, de les réorienter. Toutes les passions de l'âme se retournent vers lui, pour être purifiées.

Ces " colères " rappellent aussi la folie d'amour d'Albanio, ce désespoir agressif qui le saisit parce que Camila lui échappe. La fureur - l'amor hereos est une dimension de la psychologie amoureuse qui n'est pas étrangère à l'expérience mystique. Et dans ce dialogue que nous avons voulu surprendre entre Garcilaso et Jean de la croix, le corps n'est pas d'un côté, l'esprit de l'autre, le profane ici, et le divin là. On sort des catégories qui pourraient enfermer la poésie de Garcilaso dans les limites mondaines d'une poésie de cour, et celle de Jean de la Croix dans une dimension spirituelle qui ignore le corps, le concret

41. Cántico espiritual, 9, $\$ 4-5$.

42. Ibid., $\$ 6$.

43. Ibid., strophe 10. 
de l'expérience humaine. C'est un dialogue dans lequel le silence laisse émerger des significations implicites, fécondes, intraduisibles en langage humain, si ce n'est par le détour des images symboliques et des résonances poétiques.

Et nous revenons, pour finir, à la fontaine, qui joue un rôle si essentiel dans la deuxième églogue de Garcilaso et dans le poème mystique. Cette fontaine n'est pas seulement un lieu. Elle est cette présence muette avec laquelle tous dialoguent : Albanio, Camila, et l'épouse du poème Cántico espiritual. Silencieuse, elle leur renvoie leur propre image en un jeu constant de métamorphoses, où elle se montre comme l'âme, ou l'esprit, ou le corps. Dans la fluidité de sa course cristalline, elle est aussi le temps, et l'éternité, les abîmes de l'être, Dieu, et l'homme qui s'interroge, comme Narcisse, devant ce miroir. Comme Aristée devant Protée. 